Editorial

\title{
Inflammation and Dysmetabolism in Systemic Autoimmune Diseases
}

\author{
Antonella Afeltra $\left(\mathbb{D},{ }^{1}\right.$ Antonio Abbate, ${ }^{2}$ Gabriele Valentini, ${ }^{3}$ and Roberto Giacomelli ${ }^{4}$ \\ ${ }^{1}$ Unit of Allergology, Immunology and Rheumatology, Department of Medicine, Università Campus Bio-Medico di Roma, Rome, Italy \\ ${ }^{2}$ Virginia Commonwealth University, USA \\ ${ }^{3}$ Division of Rheumatology, Department of Precision Medicine, University of Campania "Luigi Vanvitelli", Naples, Italy \\ ${ }^{4}$ Division of Rheumatology, Department of Biotechnological and Applied Clinical Science, University of L'Aquila, L'Aquila, Italy
}

Correspondence should be addressed to Antonella Afeltra; a.afeltra@unicampus.it

Received 15 January 2019; Accepted 26 May 2019; Published 22 July 2019

Copyright (c) 2019 Antonella Afeltra et al. This is an open access article distributed under the Creative Commons Attribution License, which permits unrestricted use, distribution, and reproduction in any medium, provided the original work is properly cited.

Despite advances in pathogenic and clinical knowledge, rheumatic diseases are still burdened by high morbidity, accrual of irreversible organ damage with development of disability, and increased mortality [1-4]. In the few last decades, interest in the metabolic aspects of rheumatic diseases has gradually increased. The impact of dysmetabolism on rheumatic diseases is complex and extends from pathogenesis to clinical manifestations and potential therapeutic targets.

Metabolic syndrome (MeS) is a cluster of metabolic disorders that includes visceral adipose tissue accumulation, insulin-resistance, alteration in blood cholesterol components and apolipoproteins, and systemic inflammation [5, 6]. The incidence and prevalence of metabolic syndrome is increased in several systemic autoimmune diseases with possible impact on cardiovascular complication and damage accrual [7-9]. One of the possible links between metabolism, $\mathrm{MeS}$, and inflammation is adipokines, a group of cytokines mainly produced by adipose tissue. Consistent literature data clearly demonstrated the involvement of adipokines in autoimmunity and several systemic autoimmune diseases. In this issue, P. Ruscitti et al. deeply reviewed the role of adipokines in the atherogenesis and MeS development in patients with rheumatoid arthritis.

Interleukin-6 (IL-6) is the prototype of a molecular link between inflammation, autoimmunity, metabolism, and adipose tissue [5]. In this issue, A. Laudisio et al. analyzed the impact of olfactory dysfunction on frailty and mortality of elderly patients and demonstrated that this relation could be mediated by IL- 6 .

Particularly interesting are the implications of Western diet in rheumatic diseases. Polyunsaturated fatty acids (PUFAs) are members of the family of fatty acids, with a wide spectrum of immunological functions: $n-6$ PUFAs have predominantly proinflammatory features, while n-3 PUFAs seem to exert anti-inflammatory and proresolving properties. We recently reviewed the literature on PUFA in rheumatoid arthritis, showing that n-3-PUFA supplementation could represent an interesting therapeutic option [10]. D-Series resolvins are a product of the metabolism of n-3 PUFA. Crescent data demonstrated the involvement of $\mathrm{D}$-series resolvins and, in particular, resolvin-D1 in immune homeostasis. In general, resolvin-D1 seems to downregulate the production of proinflammatory cytokines from $\mathrm{T}$ helper 1 and $\mathrm{T}$ helper 17 lymphocytes and to promote the differentiation of $\mathrm{T}$ regulatory cells. However, only few data are available on the role of resolvins in systemic autoimmune diseases. In a paper published in this special issue, L. Navarini et al. demonstrated a marked reduction of resolvin-D1 levels in patients affected by Systemic Lupus Erythematosus (SLE) compared to the general population, especially in association with low complement levels. These findings suggest a specific role of bioactive lipids in SLE [11].

Another relevant topic in the field of relation between inflammation and metabolism is represented by bile acids. Bile acids play a pivotal role in intestinal absorption of fatty 
acids and in delivery of fatty acids to the apical membrane of enterocytes. K. Uchiyama et al. presented in this special issue a review of the available evidences on the implication of dietary lipids and fatty acids malabsorption in Crohn disease.

Overall, crescent data demonstrated the involvement of metabolism in several aspects of systemic autoimmune diseases with interesting implication for disease prevention, optimization of disease management, and drug development.

\section{Conflicts of Interest}

The editors declare that they have no conflicts of interest regarding the publication of this special issue.

\section{Antonella Afeltra Antonio Abbate Gabriele Valentini Roberto Giacomelli}

[11] L. Navarini, T. Bisogno, P. Mozetic et al., "Endocannabinoid system in systemic lupus erythematosus: first evidence for a deranged 2-arachidonoylglycerol metabolism," The International Journal of Biochemistry \& Cell Biology, vol. 99, pp. 161-168, 2018.

\section{References}

[1] R. Giacomelli, A. Afeltra, A. Alunno et al., "Guidelines for biomarkers in autoimmune rheumatic diseases - evidence based analysis," Autoimmunity Reviews, vol. 18, no. 1, pp. 93-106, 2019.

[2] F. Cacciapaglia, L. Navarini, P. Menna, E. Salvatorelli, G. Minotti, and A. Afeltra, "Cardiovascular safety of antiTNF-alpha therapies: facts and unsettled issues," Autoimmunity Reviews, vol. 10, no. 10, pp. 631-635, 2011.

[3] A. Afeltra, A. Gigante, D. P. E. Margiotta et al., "The involvement of $\mathrm{T}$ regulatory lymphocytes in a cohort of lupus nephritis patients: a pilot study," Internal and Emergency Medicine, vol. 10, no. 6, pp. 677-683, 2015.

[4] M. Vadacca, D. Margiotta, D. Sambataro et al., "BAFF/APRIL pathway in Sjögren syndrome and systemic lupus erythematosus: relationship with chronic inflammation and disease activity," Reumatismo, vol. 62, no. 4, pp. 259-265, 2010.

[5] V. Abella, M. Scotece, J. Conde et al., "Adipokines, metabolic syndrome and rheumatic diseases," Journal of Immunology Research, vol. 2014, Article ID 343746, 14 pages, 2014.

[6] L. S. Sperling, J. I. Mechanick, I. J. Neeland et al., "The CardioMetabolic Health Alliance: working toward a new care model for the metabolic syndrome," Journal of the American College of Cardiology, vol. 66, no. 9, pp. 1050-1067, 2015.

[7] D. P. E. Margiotta, F. Basta, G. Dolcini et al., "Physical activity and sedentary behavior in patients with systemic lupus erythematosus," PLoS One, vol. 13, no. 3, article e0193728, 2018.

[8] D. P. E. Margiotta, F. Basta, G. Dolcini, V. Batani, L. Navarini, and A. Afeltra, "The relation between, metabolic syndrome and quality of life in patients with systemic lupus erythematosus," PLoS One, vol. 12, no. 11, article e0187645, 2017.

[9] S. Fasano, D. P. Margiotta, L. Navarini et al., "Primary prevention of cardiovascular disease in patients with systemic lupus erythematosus: case series and literature review," Lupus, vol. 26, no. 14, pp. 1463-1472, 2017.

[10] L. Navarini, A. Afeltra, G. Gallo Afflitto, and D. P. E. Margiotta, "Polyunsaturated fatty acids: any role in rheumatoid arthritis?," Lipids in Health and Disease, vol. 16, no. 1, 2017. 


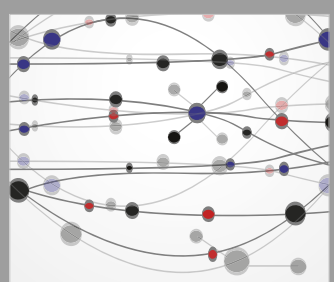

The Scientific World Journal
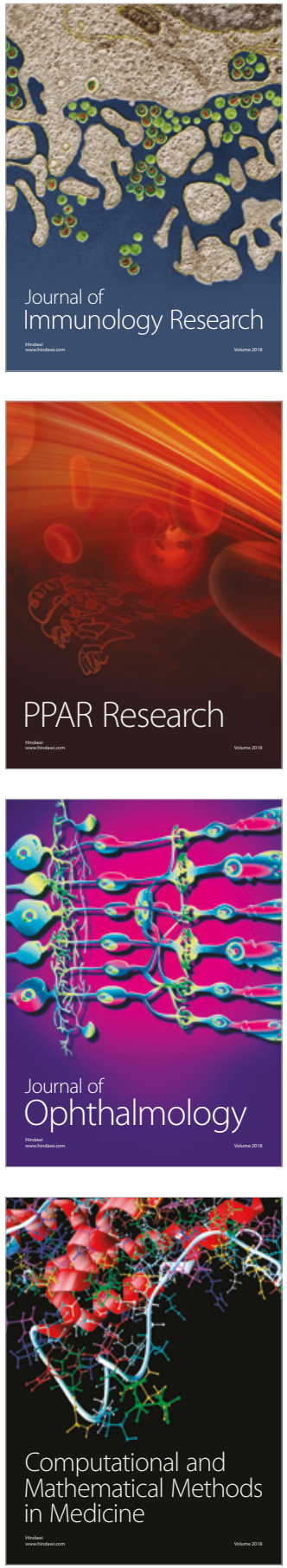

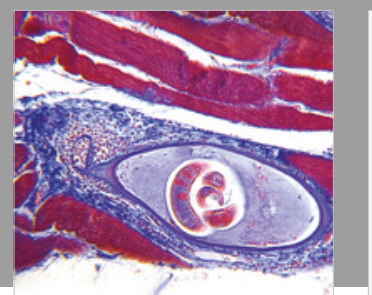

Gastroenterology Research and Practice

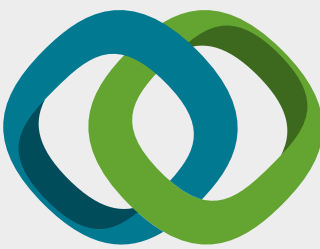

\section{Hindawi}

Submit your manuscripts at

www.hindawi.com
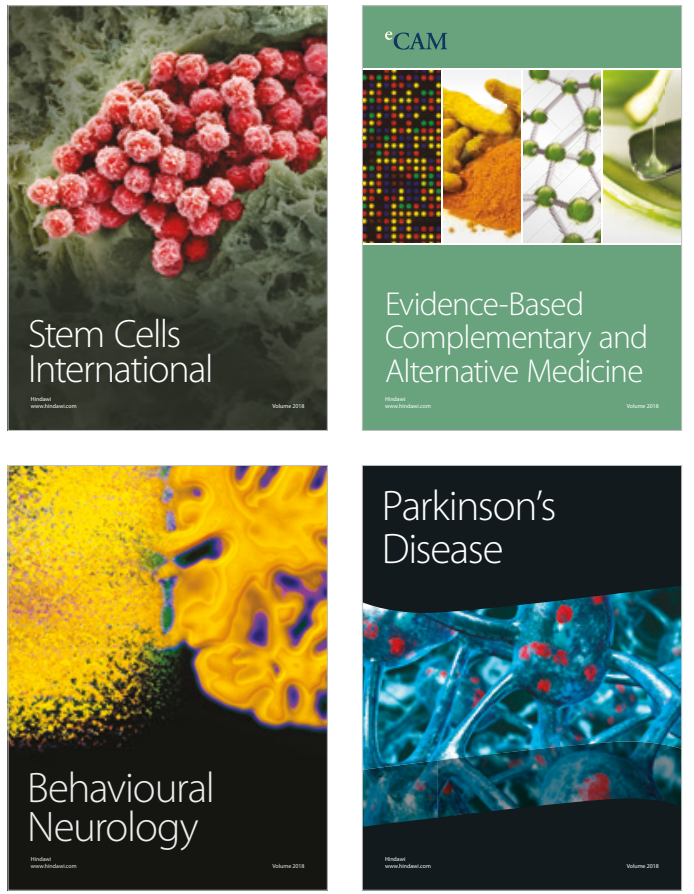

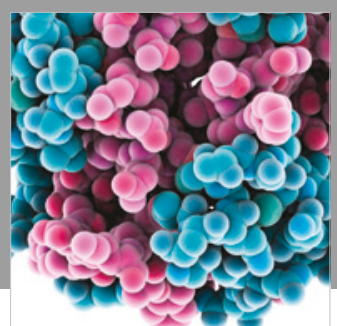

ournal of

Diabetes Research

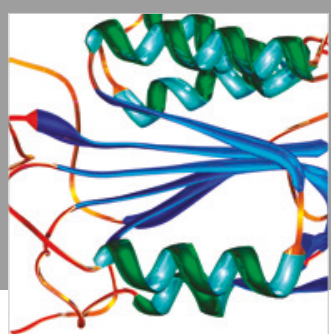

Disease Markers
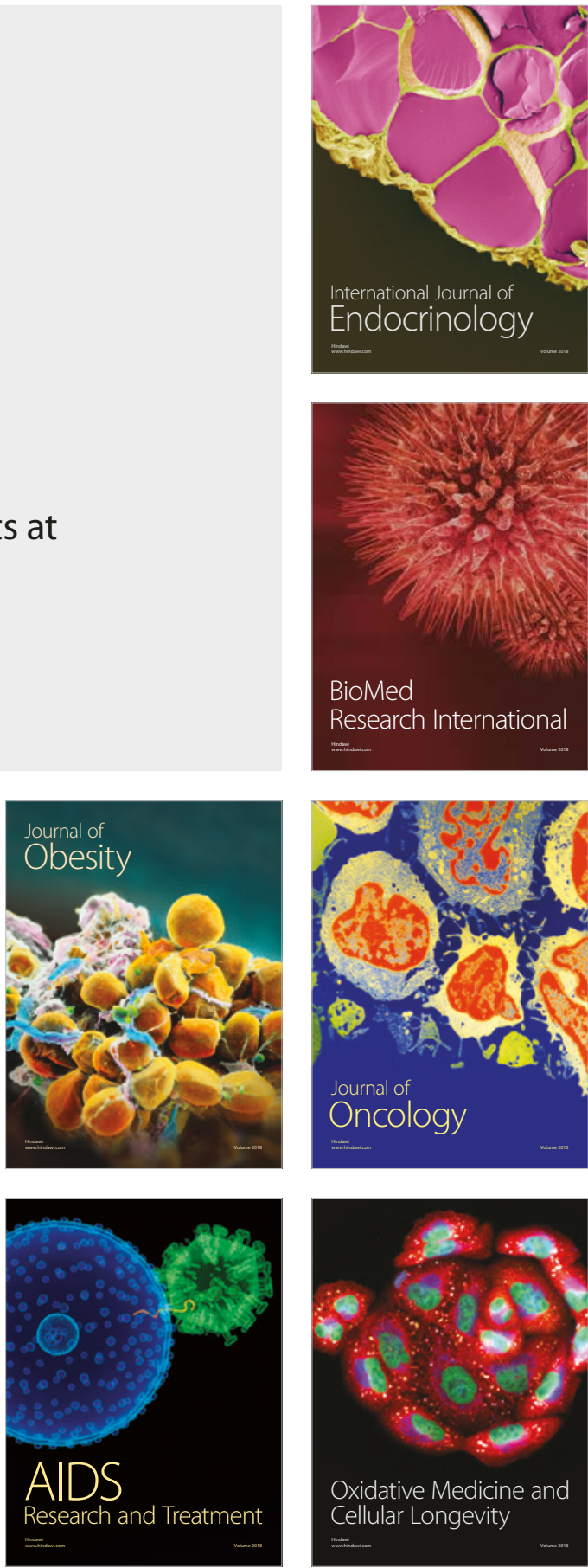